\title{
The Laparoscopic Anatomy of Rouviere's Sulcus
}

\author{
Mohamed Rabei Abdelfattah (1) \\ Department of Surgery, University of \\ Alexandria, Faculty of Medicine, \\ Alexandria, Egypt
}

Background: Laparoscopic cholecystectomy (LC) is incapacitated by occurrence of bile duct injury (BDI). Precise identification of the frequently variable biliary anatomy is important for prevention of BDI. Rouviere's sulcus received a significant interest as useful landmark to prevent BDI. This study aims to describe the frequency of Rouviere's sulcus, its various patterns, dimension and to assess its usefulness as a landmark during LC.

Methods: The study was carried on 100 consecutive cases of LC performed at our center between July 2017 and July 2018. Analysis of video recording of the laparoscopic procedure together with operative measurements was used to assess frequency, pattern, direction and various measurements of Rouviere's sulcus.

Results: Rouviere's sulcus was evident during LC in $86 \%$ of cases and the sulcus type (whether open or closed) constituted $80.2 \%$ of evident Rouviere's sulcus. The average measurement for this type was found to be about an inch in length, and less than $1 \mathrm{~cm}$ in both width and depth. Closed type of Rouviere's sulcus was significantly narrower compared to open type $(\mathrm{p}=0.00)$, while the difference in length and depth did not reach statistical significance.

Conclusion: Rouviere's sulcus is evident in majority of cases with sulcus type as its commonest pattern. Rouviere's sulcus served as a useful landmark if it was visualized and not vertically directed. It allows for safe biliary dissection through identifying the level of common bile duct in more $86 \%$ of cases.

Keywords: Rouviere's sulcus, laparoscopic cholecystectomy, bile duct injury, LC, BDI

\section{Introduction}

National Institute of Health recognized laparoscopic cholecystectomy (LC) as the new gold standard treatment for symptomatic gallstone disease. ${ }^{1}$ Consequently, LC is now one of the most frequently performed surgical procedures across the world. ${ }^{2}$ Nevertheless, LC was always incapacitated by a constant rate of bile duct injury (BDI). This significant complication is reported to occur at rate of $0.25-0.5 \%$ following $\mathrm{LC}^{3}$

Misidentification of biliary anatomy is the main reason behind the catastrophic BDI during LC. ${ }^{4}$ Therefore, precise identification of frequently variable biliary anatomy is a key factor in prevention of BDI during LC. ${ }^{5}$ Critical view of safety (CVS) is an operative strategy to prevent BDI by using various anatomical landmarks during LC. ${ }^{6}$ Rouviere's sulcus is a naturally occurring fissure on the visceral surface of the junction between right and caudate lobe. ${ }^{7}$ It received a significant interest as useful anatomical landmark to prevent BDI. ${ }^{8-10}$

BDI is occasionally encountered complication at our center. We decided to study the laparoscopic anatomy of Rouviere's sulcus and to assess its usefulness as an anatomical landmark for preventing BDI at our center. This study aims at
Correspondence: Mohamed Rabei Abdelfattah

Department of Surgery, Faculty of Medicine, AL Khartoum Square, PO Box

2II3I, Azzaritta, Alexandria, Egypt

Email Mohamad.rabie@gmail.com 
describing the frequency of Rouviere's sulcus, and its various anatomical patterns as seen during $\mathrm{LC}$ at our center.

\section{Materials and Methods}

The study was carried out on 100 consecutive cases of LC performed at our center between July 2017 and July 2018.

Approval from institutional research ethical committee was obtained before start of this study. All patients included were consented for the anonymous use of their clinical data for research purposes. Detailed demographic, clinical and operative data were collected for each case. The study was performed in accordance with the Declaration of Helsinki.

LC was carried on using the original technique described by Reddick \& Olsen, ${ }^{11}$ this technique entails the following key points: positioning of the patient and the surgical team in specific order, closed technique of creation of pneumoperitoneum, four trocars insertion, wide display of Calot's triangle and infundibular dissection first technique.

The procedure ensues by placing the patient in a supine position with both arm abducted, main surgeon and cameraman stands on the left side of the patient facing patients' right shoulder, the first assistant stands on the right side of the patient.

Creation of pneumoperitoneum begins by using a $\mathrm{CO} 2$ insufflator as a Veress needle is inserted through umbilical incision. Four trocars are then inserted, one umbilical trocar, one epigastric and two smaller trocars in the right hypochondrium, the videoscope is inserted through the umbilical trocar.

The procedure starts by laparoscopic exploration of the abdomen followed by exposure of the gallbladder. The key step in this technique entails upward-outward traction of the fundus of the gallbladder and lateral traction on the gallbladder neck to open the Calot's triangle.

Infundibular dissection first then follows to identify and dissect cystic duct and artery. While ensuring CVS prerequisites, these structures are then double clipped. The gallbladder is then dissected from its hepatic fossa and removed through the epigastric trocar.

Cases of LC performed using other techniques (e.g., French, single incision) were excluded from study. Intraoperative cholangiography is not a routine step for performance of LC at our center.

Analysis of video recording of the laparoscopic procedure together with operative measurements were used to assess frequency, pattern, direction and various measurements of RS. Terms originally described by Singh et al were used to label different patterns of the sulcus. ${ }^{12}$

Accordingly, absent Rouviere's sulcus was defined as the absence of any laparoscopic evidence of the scar including the absence of white scar just lateral to gallbladder infundibulum. Rouviere's sulcus was labeled as a scar if the laparoscopically identifiable Rouviere's sulcus was represented by superficial white scar tissue with no depth into the hepatic parenchyma immediately lateral to the gallbladder neck.

Rouviere's sulcus evident as shallow groove was termed slit type while the deep groove pattern was labeled as sulcus type it was further divided into open type when its medial end is open toward the neck of the gallbladder and closed type when it closed from its medial side. Various dimensions of the sulcus were obtained if the sulcus was found to be of the deep type only.

Dimensions of the sulcus were taken using a neonatal nasogastric tube graded in centimeters. Data collected were first expressed by descriptive statistics including Mean, range and standard deviation and data were further analyzed using $t$-test, chi-square, and Fisher's exact test. P-value of $<0.05$ was considered statistically significant.

\section{Results}

The current study includes hundred-cases of L.C performed at our center for the presence of symptomatic gallstone disease. Absent Rouviere's sulcus was encountered in $14 \%$ of cases, while it was evident in the remaining 86 cases (86\%), Rouviere's sulcus was laparoscopically evident as a sulcus in the majority of evident cases (69 cases, 80.2\%). The frequency of various types of laparoscopically evident Rouviere's sulcus is shown in Table 1. Different patterns for Rouviere's sulcus as viewed during LC are shown in Figures 1-6.

For the sulcus type of Rouviere's sulcus, the length varied between $1.4-4.6 \mathrm{~cm}$ with a mean of $2.4 \pm 0.8 \mathrm{~cm}$, on the other hand the width ranged from 0.4 to $1.1 \mathrm{~cm}$ with a mean of $0.8 \pm 0.15 \mathrm{~cm}$. Finally, the depth varied between 0.5 and $0.9 \mathrm{~cm}$ with a mean of $0.7 \pm 0.13 \mathrm{~cm}$. In summary, the sulcus type of Rouviere's sulcus was about an inch in length, less than $1 \mathrm{~cm}$ in both width, and depth. Except for the depth, the mean values for Rouviere's sulcus dimensions did not differ significantly between closed and open type of Rouviere's sulcus, as shown in Table 2.

In cases of visualized Rouviere's sulcus, LC was converted to open procedure in four cases $(4.7 \%)$. For the remaining 82 cases three case were excluded due to the 
Table I Frequency, and Percentage of Various Types of Laparoscopically Evident Rouviere's Sulcus

\begin{tabular}{|c|c|c|c|c|c|c|}
\hline \multicolumn{2}{|c|}{ Type of Rouviere's Sulcus } & \multirow[t]{2}{*}{ Number of Cases } & \multirow[t]{2}{*}{ Percentage* } & \multicolumn{3}{|c|}{ Direction } \\
\hline & & & & Oblique & Horizontal & Vertical \\
\hline \multicolumn{2}{|l|}{ Scar } & 6 & $7 \%$ & 3 & I & 2 \\
\hline \multicolumn{2}{|l|}{ Slit } & 11 & $12.8 \%$ & 7 & 3 & I \\
\hline \multirow[t]{2}{*}{ Sulcus } & Open & $53(76.8 \%)^{* *}$ & $61.6 \%$ & 48 & 5 & 0 \\
\hline & Closed & $16(23.2 \%)^{* *}$ & $18.6 \%$ & 12 & 4 & 0 \\
\hline \multicolumn{2}{|l|}{ Total } & 86 & $100 \%$ & 70 & 13 & 3 \\
\hline
\end{tabular}

Notes: *Percentage out of total number of laparoscopically evident Rouviere's sulcus. **Percentage out of total number of sulcus type.

presence of vertically directed Rouviere's sulcus. After complete dissection in the remaining seventy- nine cases, the plane for Rouviere's Sulcus was found to be higher than the level for common bile duct in 68 patients (86.1\%). It is therefore clear that keeping the dissection above the level of transversely directed Rouviere's sulcus can prevent injury to common bile duct in majority of our patients.

On the other hand, only one patient was converted to open procedure in the group of non-visualized

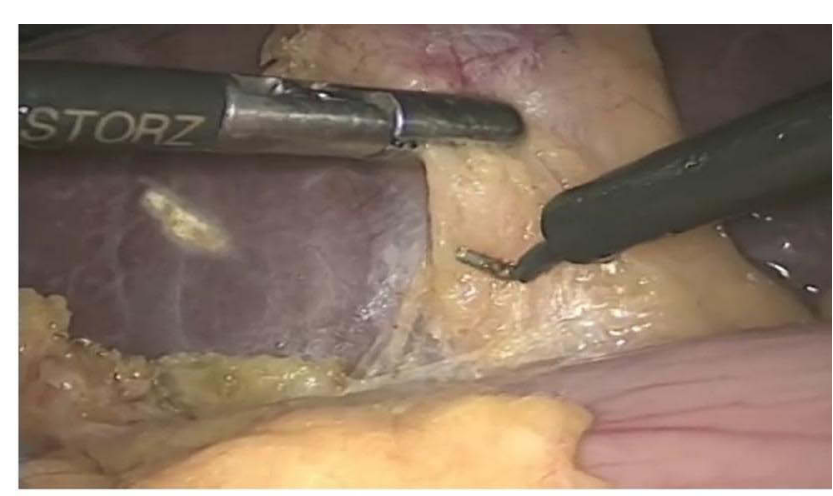

Figure I Absellt Rouviere's sulcus.

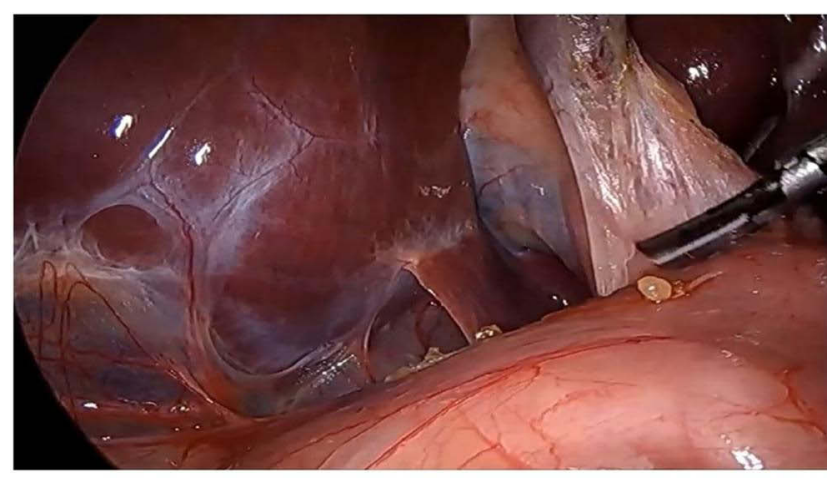

Figure 2 Rouviere's sulcus of scar type.
Rouviere's sulcus (6.3\%). Conversion rate did not differ between cases with visualized or absent Rouviere's sulcus, $\mathrm{P}=1$. Whether Rouviere's sulcus was visualized or absent, none of LC patients was complicated by BDI during this study.

\section{Discussion}

BDI is catastrophic complication of LC with profound medical and financial sequelae. Thus, prevention of BDI is crucial especially in the light of the huge number of LC

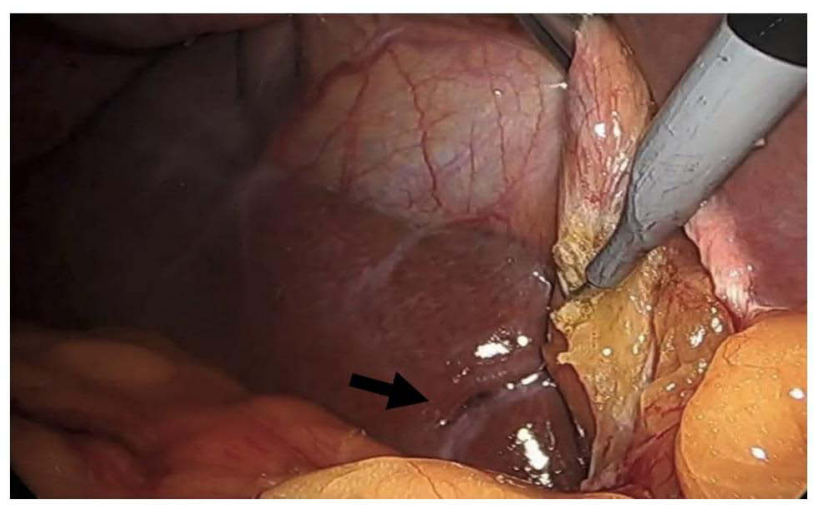

Figure 3 Slit type Rouviere's sulcus (Black arrow).

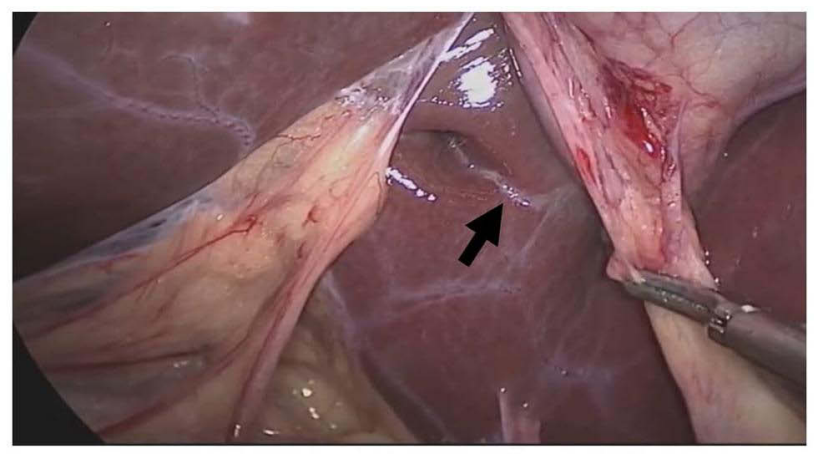

Figure 4 Closed type of Rouviere's sulcus (black arrow is showing closed medial end). 


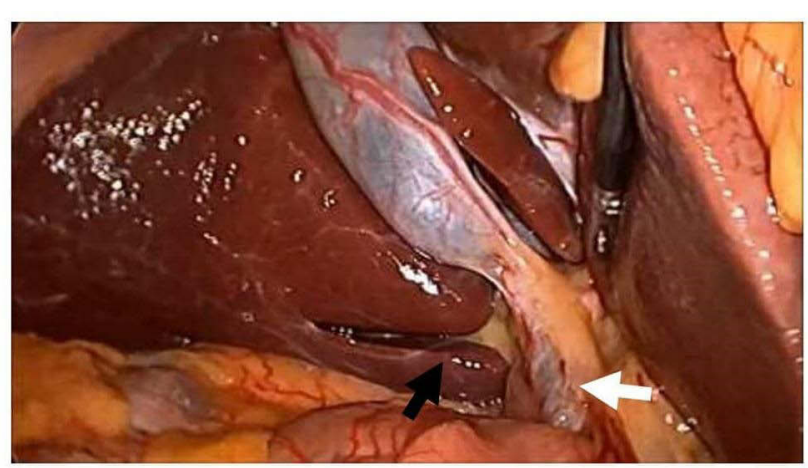

Figure 5 Open type Rouviere's sulcus (Black arrmv: opell medial elld of Rouviere's sulcus, white arrmv: Common bile duct).

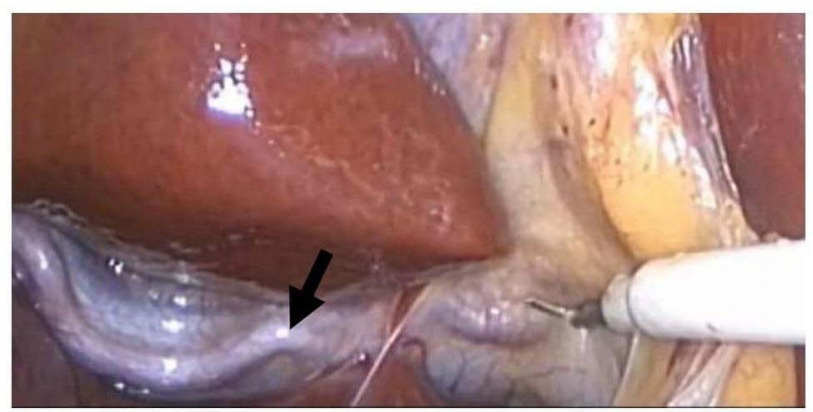

Figure 6 Close laparoscopic view of open type Rouviere's sulcus with right hepatic artery visualized in its floor (black arrow).

procedures performed. The CVS was described to provide a tool for surgeons to avoid misinterpretation of biliary anatomy. ${ }^{6} \mathrm{CVS}$ was found to decrease and - sometime to eliminate - the incidence of BDI. ${ }^{13}$

Nevertheless, CVS can be affected by severe inflammatory process involving gallbladder and nearby Calot's triangle. This highlights the need for anatomical landmark that permits safe dissection during LC while being spared from distortion by inflammation and fibrosis. In this context, Rouviere's sulcus serves as ideal anatomical landmark during LC. ${ }^{14}$

Rouviere's sulcus was first described by Rouviere back in 1924 as a furrow or fissure related to the caudate lobe. ${ }^{15}$ The term "Rouviere's sulcus" was coined by the famous French hepatobiliary surgeon "Claude Couinaud." Nomenclature of the different patterns of Rouviere's sulcus was not standardized by anatomist. The current terms used to describe patterns of Rouviere's sulcus stems from laparoscopic studies where the need is maximum for preventing BDI during LC. ${ }^{12}$

The described incidence and dimensions of visualized Rouviere sulcus in the current study are very similar to what was reported in the literature. There are minor variations in the reported incidence of Rouviere's sulcus in the medical literature. It was reported to be visualized in 75 $90 \%$ of patients.

The current study matches the abovementioned range. Thapa et $\mathrm{al}^{16}$ reported visualized Rouviere's sulcus in of $75 \%$ of patients, Dahmane et $\mathrm{al}^{8}$ reported an incidence of $82 \%$, while Peti et $\mathrm{al}^{7}$ described an incidence of $80 \%$. The highest reported incidence of Rouviere's sulcus (> 90\% visualization rate) was reported by Singh et $\mathrm{al}^{12}$ and Kumar et al. ${ }^{17}$ In a recent meta-analysis of 23 anatomical or laparoscopic studies, Cheruiyot et al ${ }^{18}$ reported an overall incidence of $83 \%$. These minor variations are attributed to different degrees of including the scar type of Rouviere's sulcus in these studies. The reported dimensions of Rouviere's sulcus in the current study match what was described by Singh et al. ${ }^{12}$ Visualization of Rouviere's sulcus is very common and easy during LC, Kumar et $\mathrm{al}^{17}$ attributed this to $\mathrm{CO} 2$ insufflation superior illumination and excellent image quality.

The current study demonstrated the usefulness of Rouviere's sulcus in identifying the level of common bile duct during LC. Its main advantage in this regard is being spared from fibrosis which can otherwise involve the Calot's triangle. It also revealed that the level of common bile duct was below the level transversely directed Rouviere's sulcus in more than $86 \%$ of cases.

Similarly, Jha et al ${ }^{19}$ reported that the level for common bile duct was below the level of Rouviere's sulcus in about $80 \%$ of the cases whenever Rouviere's sulcus was visible. In the same context, Kumar et $\mathrm{al}^{17}$ stated that identification of Rouviere's sulcus during LC precludes major complications.

\section{Conclusions}

Rouviere's sulcus was found to be a useful and commonly visualized anatomical landmarks in $86 \%$ of cases. Sulcus

Table 2 Comparison of Mean Length, Width, and Depth for Open vs Closed Type of Rouviere's Sulcus with Their Respective P value

\begin{tabular}{|l|c|c|c|c|c|c|}
\hline Sulcus Type & Mean Length & P value & Mean Width & P value & Mean Depth & P value \\
\hline Open type & $2.6 \pm 0.8 \mathrm{~cm}$ & $P=0.09$ & $\begin{array}{l}0.9 \pm 0.2 \mathrm{~cm} \\
0.6 \pm 0.1 \mathrm{~cm}\end{array}$ & $P=0.00$ & $\begin{array}{c}0.8 \pm 0.2 \mathrm{~cm} \\
0.7 \pm 0.1 .5 \mathrm{~cm}\end{array}$ & $P=0.07$ \\
Closed type & $2.2 \pm 0.9 \mathrm{~cm}$ & & & & \\
\hline
\end{tabular}


type is the commonest pattern for its occurrence. The described incidence and dimensions of visualized Rouviere sulcus in the current study are very similar to what was reported in the literature. Rouviere's sulcus served as a useful landmark if it was visualized and not vertically directed.

\section{Abbreviations}

BDI, bile duct injury; CVS, critical view of safety; LC, laparoscopic cholecystectomy.

\section{Ethical Approval}

Approval of this research work was obtained from ethical research committee of Faculty of Medicine, Alexandria University before starting the study.

\section{Consent for Publication}

All patients consented for anonymous use of their clinical data and anonymized intraoperative images for research purposes.

\section{Funding}

The author declares that no funding was received.

\section{Disclosure}

The author reported no conflicts of interest for this work.

\section{References}

1. Soper NJ, Stockmann PT, Dunnegan DL, Ashley SW. Laparoscopic cholecystectomy. The new 'gold standard'? Arch Surg. 1992;127 (8):917-921. doi:10.1001/archsurg.1992.01420080051008

2. Gaillard M, Tranchart H, Lainas $P$, et al. New minimally invasive approaches for cholecystectomy: review of the literature. World J Gastrointest Surg. 2015;7:243-248. doi:10.4240/wjgs.v7.i10.243

3. Vollmer CMM, Callery MP. Biliary injury following laparoscopic cholecystectomy: why still a problem? Gastroenterology. 2007;133:1039-1041. doi:10.1053/j.gastro.2007.07.041

4. Pucher PH, Brunt LM, Fanelli RD, Asbun HJ, Aggarwal R. SAGES expert Delphi consensus: critical factors for safe surgical practice in laparoscopic cholecystectomy. Surg Endosc. 2015;29(11):3074-3085. doi:10.1007/s00464-015-4079-z
5. Shang P, Liu B, Li X, Miao J, Lv R, Guo W. A practical new strategy to prevent bile duct injury during laparoscopic cholecystectomy. A single-center experience with 5539 cases. Acta Cir Bras. 2020;35(6): e202000607. doi:10.1590/s0102-865020200060000007

6. Strasberg SM. Rationale and use of the critical view of safety in laparoscopic cholecystectomy. J Am Coll Surg. 2010;211(1):13 2-138. doi:10.1016/j.jamcollsurg.2010.02.053

7. Peti N, Moser MA. Graphic reminder of Rouviere's sulcus: a useful landmark in cholecystectomy. ANZ J Surg. 2012;82(5):367-368. doi:10.1111/j.1445-2197.2012.06032.x

8. Dahmane R, Morjane A, Starc A. Anatomy and surgical relevance of Rouviere's sulcus. Sci World J. 2013;2013:254287. doi:10.1155/ 2013/254287

9. Zubair M, Habib L, Memon F. Rouviere's sulcus: a guide to safe dissection in laparoscopic cholecystectomy. Pak J Surg. 2009;25: $119-121$

10. Lockhart S, Singh-Ranger G. Rouviere's sulcus-aspects of incorporating this valuable sign for laparoscopic cholecystectomy. Asian J Surg. 2018;41(1):1-3. doi:10.1016/j.asjsur.2016.07.012

11. Reddick EJ, Olsen D, Spaw A, et al. Safe performance of difficult laparoscopic cholecystectomies. Am J Surg. 1991;161(3):377-381. doi:10.1016/0002-9610(91)90601-9

12. Singh M, Prasad N. The anatomy of Rouviere's sulcus as seen during laparoscopic cholecystectomy: a proposed classification. J Minim Access Surg. 2017;13(2):89-95. doi:10.4103/0972-9941.201731

13. Bulent K, Fersahoglu MM, Killic F, Onur E, Memisoglu K. Importance of critical view of safety in laparoscopic cholecystectomy: a survey of 120 serial patients, with no incidence of complications. Ann Hepatobiliary Pancreat Surg. 2017;21:17-20. doi:10.14701/ahbps.2017.21.1.17

14. Hugh TB, Kelly MD, Mekisic A. Rouviere's sulcus: a useful landmark in laparoscopic cholecystectomy. Br J Surg. 1997;84:12 53-1254.

15. Rouviere H. On the configuration and the significance of the groove of the caudate process. Newsl Mem Anat Soc Paris. 1924;94: 355-358.

16. Thapa PB, Maharjan DK, Tamang TY, Shrestha SK. Visualisation of Rouviere's sulcus during laparoscopic cholecystectomy. JNMA $J$ Nepal Med Assoc. 2015;53(199):188-191. doi:10.31729/jnma. 2789

17. Kumar A, Shah R, Pandit N, Sah SP, Gupta RK. Anatomy of Rouviere's sulcus and its association with complication of laparoscopic cholecystectomy. Minim Invasive Surg. 2020;2020:395 6070.

18. Cheruiyot I, Nyaanga F, Kipkorir V, et al. The prevalence of the Rouviere's sulcus: a meta-analysis with implications for laparoscopic cholecystectomy. Clin Anat. 2021;34(4):556-564. doi:10.1002/ ca. 23605

19. Jha AK, Dewan R, Bhaduria K. Importance of Rouviere's sulcus in laparoscopic cholecystectomy. Ann Afr Med. 2020;19(4):274-277. doi:10.4103/aam.aam_4_20
Open Access Surgery

\section{Publish your work in this journal}

Open Access Surgery is an international, peer-reviewed, open access journal that focuses on all aspects of surgical procedures and interventions. Patient care around the peri-operative period and patient outcomes post-surgery are key topics for the journal. All grades of surgery from minor cosmetic interventions to major surgical procedures are covered. Novel techniques and the utilization of new

Submit your manuscript here: https://www.dovepress.com/open-access-surgery-journa instruments and materials, including implants and prostheses that optimize outcomes constitute major areas of interest. The manuscript management system is completely online and includes a very quick and fair peer-review system, which is all easy to use. Visit http://www.dovepress.com/testimonials.php to read real quotes from published authors. 